\title{
Abnormalities of endothelial function in patients with predialysis renal failure
}

\author{
J Thambyrajah, M J Landray, F J McGlynn, H J Jones, D C Wheeler, J N Townend
}

\begin{abstract}
Background-Endothelial dysfunction plays an important role in the development of atherosclerotic vascular disease, which is the leading cause of mortality in patients with chronic renal failure.

Objective-To examine the relation between predialysis renal failure and endothelial function. Design-Two groups were studied: 80 patients with non-diabetic chronic renal failure and 26 healthy controls, with similar age and sex distributions. Two indices of endothelial function were assessed: high resolution ultrasonography to measure flow mediated endothelium dependent dilatation of the brachial artery following reactive hyperaemia, and plasma concentration of von Willebrand factor. Endothelium independent dilatation was also assessed following sublingual glyceryl trinitrate. The patients were divided into those with and without overt atherosclerotic vascular disease.

Results-Although patients with chronic renal failure had significantly impaired endothelium dependent dilatation compared with controls (median (interquartile range), $2.6 \%(0.7 \%$ to $4.8 \%$ ) v $6.5 \%(4.8 \%$ to $8.3 \%) ; \mathrm{p}<0.001)$ and increased von Willebrand factor $(254$ (207 to 294$)$ $v 106$ (87 to 138) iu/dl; $\mathrm{p}<0.001$ ), there was no difference between renal failure patients with and without atherosclerotic vascular disease. Within the chronic renal failure group, endothelium dependent dilatation and von Willebrand factor were similar in patients in the upper and lower quartiles of glomerular filtration rate $(2.7 \%(0.7 \%$ to $6.7 \%) v 2.8 \%(1.1 \%$ to $5.0 \%)$; and 255 (205 to 291) v 254 (209 to 292) iu/dl, respectively). Endothelium independent dilatation did not differ between the renal failure or control groups and was also similar in patients with renal failure irrespective of the degree of renal failure or the presence of atherosclerotic vascular disease. Conclusions-Endothelial function is abnormal in chronic renal failure, even in patients with mild renal insufficiency and those without atherosclerotic vascular disease, suggesting that uraemia may directly promote the development of atherosclerosis early in the progression of chronic renal failure.
\end{abstract}

(Heart 2000;83:205-209)

Keywords: atherosclerosis; endothelial function; flow mediated dilatation; renal failure

Advances in the provision of renal replacement therapy have reduced deaths from uraemia but have revealed the clinical significance of atherosclerotic vascular disease in patients with chronic renal failure. Cardiovascular disease is the leading cause of mortality in these patients, with a 16 -fold to 19 -fold increased risk of myocardial ischaemia and infarction compared with control subjects. ${ }^{1-3}$ This accounts for much of the $70-80 \%$ reduction in life expectancy of patients with end stage renal disease. ${ }^{4}$

The response to injury hypothesis proposes that endothelial injury and dysfunction are the primary processes in the pathogenesis of atherosclerosis because they result in lipid accumulation, smooth muscle proliferation, and a tendency to vasospasm and thrombosis. ${ }^{5}$ Endothelial function can be assessed noninvasively using high resolution ultrasound to measure flow mediated endothelium dependent dilatation of the brachial artery, and also by determining plasma concentrations of circulating von Willebrand factor. ${ }^{6}$ Impaired endothelium dependent dilatation has been demonstrated in asymptomatic children and young adults with established risk factors for atherosclerosis, and there is a close correlation between brachial and coronary artery endothelial dysfunction. ${ }^{6-8}$ Von Willebrand factor is an endothelium derived protein that is involved in the regulation of haemostasis. ${ }^{9}$ Raised plasma concentrations of circulating von Willebrand factor are associated with endothelial injury and predict the development and progression of cardiovascular disease. ${ }^{10}{ }^{11}$ Reduced endothelium dependent dilatation and increased plasma von Willebrand factor levels have been demonstrated in patients with renal failure on maintenancehaemodialysis. ${ }^{12-14}$ However, endothelial function may be altered not only by the process of dialysis itself but also by asymptomatic atherosclerotic disease which may be present in patients with end stage renal disease. $^{15}$

This study was designed to assess endothelial function at an earlier stage of progressive renal failure in predialysis patients; to examine the relation between renal failure and endothelial function; and to determine whether abnormalities of endothelial function are related to the presence of clinically evident atherosclerotic vascular disease in patients with chronic renal failure.

\section{Methods}

SUBJECTS

Eighty patients with chronic renal failure (serum creatinine $>130 \mu \mathrm{mol} / \mathrm{l}$ ) were recruited 
Table 1 Definitions of clinical criteria for the presence of atherosclerotic vascular disease and risk factors

\begin{tabular}{|c|c|}
\hline Clinical criteria & Definitions \\
\hline Coronary artery disease & $\begin{array}{l}\text { Any one of the following: documented history of myocardial } \\
\text { infarction confirmed by Q waves on a } 12 \text { lead ECG; typical } \\
\text { anginal chest pain confirmed by evidence of inducible ischaemia } \\
\text { (exercise ECG, or myocardial perfusion scan); angiographic } \\
\text { evidence; coronary revascularisation procedure }\end{array}$ \\
\hline Cerebrovascular disease & Documented episode of sudden onset neurological deficit \\
\hline Peripheral vascular disease & $\begin{array}{l}\text { Any of the following: documented history of intermittent } \\
\text { claudication or abdominal aortic aneurysm; angiographic } \\
\text { evidence; peripheral artery revascularisation procedure; } \\
\text { amputation for vascular disease }\end{array}$ \\
\hline Renovascular disease & Angiographic evidence \\
\hline Smoking history & Subjects who had smoked in the last year \\
\hline Hypertension & $\begin{array}{l}\text { Subjects on antihypertensive treatment, or with a systolic blood } \\
\text { pressure } \geqslant 160 \mathrm{~mm} \mathrm{Hg} \text { or a diastolic blood pressure } \geqslant 90 \mathrm{~mm} \\
\mathrm{Hg}\end{array}$ \\
\hline Hypercholesterolaemia & $\begin{array}{l}\text { Subjects receiving lipid lowering treatment or with fasting plasma } \\
\text { total cholesterol }>6.5 \mathrm{mmol} / 1\end{array}$ \\
\hline Family history & Presence of vascular disease in a first degree relative $<65$ years \\
\hline
\end{tabular}

from the predialysis clinic at the Queen Elizabeth Hospital, Birmingham. There were 58 men and 22 women, with a median (interquartile range) age of 65 (56 to 71 ) years. Twenty six healthy controls with no history of vascular disease (61 (51 to 72 ) years; 17 men, nine women) were recruited from a nearby general practice. Subjects with diabetes mellitus were excluded.

STUDY PROTOCOL

The study was approved by the South Birmingham local research ethics committee, and written informed consent was obtained from all participants. Following the discontinuation of vasoactive drugs for 18 hours and a 12 hour fast, all subjects underwent a brachial artery ultrasound scan. Subjects were screened by a standardised doctor administered questionnaire, using clinical criteria defined prospectively (table 1), for the presence of atherosclerotic vascular disease and risk factors. The answers were confirmed by a review of the hospital case notes. After a 10 minute period of rest, blood pressure was recorded twice in the sitting position using a standard sphygmomanometer, and fasting blood samples were taken for estimation of serum creatinine and lipid profile. The glomerular filtration rate was calculated from the formula of Cockcroft and Gault. ${ }^{16}$ Circulating plasma concentrations of von Willebrand factor were measured using enzyme linked immunosorbent assay kits (Department of Rheumatology, University of Birmingham, Birmingham, UK).

HIGH RESOLUTION ULTRASONOGRAPHY OF THE BRACHIAL ARTERY

The ultrasound procedure was performed according to the method of Celermajer et al. ${ }^{6}$ In healthy arteries, reactive hyperaemia following transient occlusion increases shear stress, which results in vasodilatation mediated by endothelium derived nitric oxide. ${ }^{118}$ Endothelium independent dilatation, a reflection of vascular smooth muscle function, can be assessed by measuring changes in the brachial artery diameter following sublingual administration of the nitric oxide donor glyceryl trinitrate.
Subjects were kept supine in a stable room temperature between $20-25^{\circ} \mathrm{C}$, with their right arm comfortably immobile in the extended position to allow access to the brachial artery. None of the subjects studied had an arteriovenous fistula. A single investigator performed all imaging and analysis. A B mode scan was obtained of the right brachial artery in cross section between $5 \mathrm{~cm}$ to $12 \mathrm{~cm}$ proximal to the antecubital fossa, using a $7.5 \mathrm{MHz}$ phased array transducer attached to a Sigma 44 HVD system (Kontron Instruments, Montigny le Bretonneux, France). After optimal transducer positioning, a resolution box function was used to magnify the images. Depth and gain settings were adjusted to maximise the definition of anterior and posterior media to intima interfaces, which were used to demarcate the brachial artery diameter. This diameter was calculated as the average of measurements made during four cardiac cycles, incident with the $\mathrm{R}$ wave of the electrocardiograph trace. All measurements were recorded on super VHS videotape for subsequent off line analysis.

Each study was composed of artery diameter measurements as follows: (1) first baseline after 10 minute period of rest to allow acclimatisation; (2) endothelium dependent dilatation 60 to 90 seconds after the response to reactive hyperaemia, induced by the sudden deflation of a pneumatic cuff placed on the ipsilateral forearm and inflated to a pressure $100 \mathrm{~mm} \mathrm{Hg}$ above systolic for five minutes; (3) second baseline after further 10 minute rest period to allow vessel recovery; (4) endothelium independent dilatation four minutes after sublingual administration of $800 \mu \mathrm{g}$ glyceryl trinitrate spray.

At the end of each rest period the baseline velocity was assessed by Doppler ultrasonography. The average baseline diameter and blood flow velocity was calculated from the first and second baseline recordings. The peak increase in blood flow velocity in response to reactive hyperaemia was recorded as the maximum velocity in a single cardiac cycle within the first 15 seconds after cuff deflation and was expressed as a percentage of the average baseline velocity. This was used as a quantitative estimate of reactive hyperaemia. Endothelium dependent and independent dilatation were expressed as the percentage change in the brachial artery diameter from baseline following reactive hyperaemia and sublingual glyceryl trinitrate, respectively.

\section{REPEATABILITY}

Intraobserver variability was calculated, based on 17 subjects. The coefficients of variation for baseline diameter, endothelium dependent dilatation, and endothelium independent dilatation were $2.1 \%, 3.7 \%$, and $2.9 \%$, respectively.

STATISTICAL ANALYSIS

Data were analysed using SPSS for Windows 9.0. Medians and interquartile ranges were used to describe continuous variables. The distribution of discrete and continuous variables between groups was compared using $\chi^{2}$ and 
Table 2 Baseline characteristics of the renal failure and control groups

\begin{tabular}{lll}
\hline & Control & Renal failure \\
\hline Number & 26 & 80 \\
Age (years) & $61(51$ to 72$)$ & $65(56$ to 71$)$ \\
Sex $(\mathrm{M}: \mathrm{F})$ & $17: 9$ & $58: 22$ \\
Smoking history & 13 & 49 \\
Family history & 8 & 30 \\
Hypertension & 12 & $72^{\star \star \star}$ \\
Hypercholesterolaemia & 2 & $26^{\star \star}$ \\
BMI $\left(\mathrm{kg} / \mathrm{m}^{2}\right)$ & $25.8(24.6$ to 29.1$)$ & $27.2(24.3$ to 29.9$)$ \\
Creatinine $(\mu$ mol/1) & $96(90$ to 107$)$ & $245(188 \text { to } 325)^{\star \star \star}$ \\
GFR $(\mathrm{ml} /$ min) & $78.3(62.3$ to 93.1$)$ & $28.9(20.3 \text { to } 37.6)^{\star \star \star}$ \\
Baseline diameter $(\mathrm{cm})$ & $0.48(0.39$ to 0.52$)$ & $0.51(0.45 \text { to } 0.56)^{\star}$ \\
Increase in velocity $(\%)$ & $208(176$ to 229$)$ & $196(169$ to 218$)$ \\
\hline
\end{tabular}

Continuous variables are expressed as median (interquartile range). Significance was assessed using $\chi^{2}$ and Mann-Whitney tests as appropriate.

${ }^{\star} \mathrm{p}<0.05 ;{ }^{\star \star} \mathrm{p}<0.01 ;{ }^{\star \star \star} \mathrm{p}<0.001$.

$\mathrm{BMI}$, body mass index; GFR, glomerular filtration rate.

Mann-Whitney tests. The Kruskal-Wallis test was used to compare continuous variables by quartiles of calculated glomerular filtration rate. Multiple regression analyses were used to correct for differences in baseline diameter and known cardiovascular risk factors. The test results are presented as two tailed values and significance was inferred at $\mathrm{p}<0.05$.

\section{Results}

COMPARISON OF RENAL FAILURE AND CONTROL GROUPS

The baseline characteristics for the renal failure and control groups are shown in table 2. The significant differences, other than renal function, were the higher prevalence of hypertension and hypercholesterolaemia and the larger median baseline brachial artery diameter in the renal failure group.

Differences in endothelial function between patients and control are illustrated in fig 1 . Endothelium dependent dilatation was significantly impaired in patients with renal failure compared with controls $(2.6 \%(0.7 \%$ to $4.8 \%)$ v $6.5 \%(4.8 \%$ to $8.3 \%) ; \mathrm{p}<0.001)$. This difference remained significant after correction for the presence of vascular risk factors and for baseline diameter. Analysis of the crude data also suggested a significant impairment of endothelium independent dilatation in patients with renal failure $(9.9 \%$ ( $7.8 \%$ to $13.8 \%) v$ $14.5 \%(10.6 \%$ to $19.0 \%))$ but this was not significant after correction for differences in baseline diameter. Plasma von Willebrand factor concentration was significantly raised in the patients with renal failure compared with the controls (254 (207 to 294) v 106 (87 to 138 ) $\mathrm{iu} / \mathrm{dl} ; \mathrm{p}<0.001)$, a difference that remained after correction for vascular risk factors.

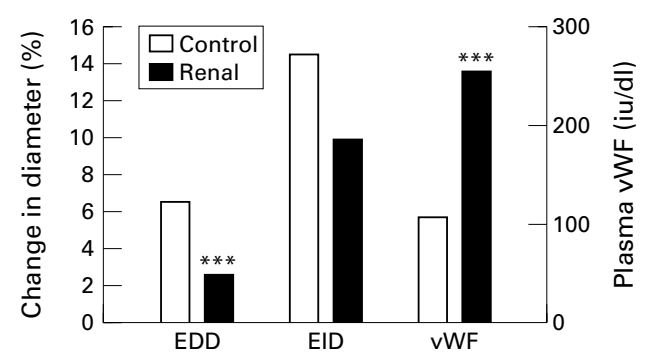

Figure 1 Endothelium dependent and independent dilatation of the brachial artery and von Willebrand factor concentration in the renal failure and control groups. EDD, endothelium dependent dilatation; EID, endothelium independent dilatation; vWF, von Willebrand factor; $\star \star \star p<0.001$ after controlling for differences in the baseline characteristics.

ENDOTHELIAL FUNCTION ACCORDING TO DEGREE OF RENAL IMPAIRMENT

The renal failure group was divided by the calculated glomerular filtration rate into quartiles with declining renal function from the first to the fourth quartiles. There were no significant differences in age, sex distribution, body mass index, smoking and family history, prevalence of hypertension and hypercholesterolaemia, and the presence of vascular disease between the four quartiles. The results for endothelium dependent dilatation and endothelium independent dilatation of the brachial artery and plasma von Willebrand factor concentration are shown in table 3. The baseline diameter and increase in velocity were similar across the quartiles. There was no significant difference in endothelium dependent dilatation, endothelium independent dilatation, or plasma von Willebrand factor concentrations between the quartiles of calculated glomerular filtration rate in patients with renal failure.

COMPARISON OF THE RENAL FAILURE PATIENTS WITH AND WITHOUT CLINICALLY EVIDENT ATHEROSCLEROTIC VASCULAR DISEASE Clinically evident atherosclerotic vascular disease was diagnosed in 29 of the renal failure patients: coronary artery disease $(n=22)$, peripheral vascular disease $(n=3)$, cerebrovascular disease $(n=3)$, and renovascular disease $(n=1)$. This group was significantly older than those without atherosclerotic vascular disease (66 (62 to 72 ) v 61 (47 to 71 ) years; $p<0.05)$ but there were no significant differences in other baseline characteristics between these two groups. After correction for age, there was no difference in endothelium dependent dilatation between renal failure patients with and without atherosclerotic vascular disease $(1.6 \%(0.3 \%$ to $3.4 \%)$ v $3.8 \%$ $(1.4 \%$ to $5.2 \%))$. Similarly, there was no

Table 3 Endothelium dependent and independent dilatation of the brachial artery and von Willebrand factor concentration according to degree of renal impairment

\begin{tabular}{|c|c|c|c|c|c|}
\hline & Controls & First quartile & Second quartile & Third quartile & Fourth quartile \\
\hline GFR (ml/min) & $78(62$ to 93$)$ & $49(46$ to 54$)$ & $32(31$ to 36$)$ & $24(22$ to 27$)$ & $16(14$ to 18$)$ \\
\hline Baseline diameter $(\mathrm{cm})$ & $0.48(0.39$ to 0.52$)$ & $0.51(0.45$ to 0.55$)$ & $0.53(0.47$ to 0.57$)$ & $0.50(0.45$ to 0.52$)$ & $0.51(0.46$ to 0.54$)$ \\
\hline Increase in velocity (\%) & 208 (176 to 229$)$ & $206(190$ to 225$)$ & $202(180$ to 218$)$ & $167(151$ to 215$)$ & 178 (169 to 213$)$ \\
\hline EDD $(\%)$ & $6.5(4.8$ to 8.3$)$ & $2.7(0.7$ to 6.7$)$ & $2.3(0.1$ to 4.8$)$ & $1.9(1.5$ to 4.3$)$ & $2.8(1.1$ to 5.0$)$ \\
\hline EID (\%) & $14.5(10.6$ to 19.0$)$ & $11.0(8.1$ to 13.4$)$ & $9.1(6.2$ to 11.0$)$ & $9.8(8.8$ to 16.8$)$ & $9.8(7.6$ to 14.6$)$ \\
\hline $\mathrm{vWF}(\mathrm{iu} / \mathrm{dl})$ & $106(87$ to 138$)$ & 255 (205 to 291$)$ & $223(185$ to 276$)$ & $273(223$ to 298$)$ & $254(209$ to 292$)$ \\
\hline
\end{tabular}

Results are expressed as median (interquartile range). Significance was assessed with the Kruskal-Wallis test.

EDD, endothelium dependent dilatation; EID, endothelium independent dilatation; GFR, glomerular filtration rate; vWF, von Willebrand factor. 
significant difference in either endothelium independent dilatation or plasma von Willebrand factor concentration between renal failure patients with and without atherosclerotic vascular disease $(9.9 \%(7.1 \%$ to $13.0 \%)$ v $10.0 \%$ ( $8.4 \%$ to $14.7 \%$ ), and 254 (227 to 294) v 246 (201 to 293 ) iu/dl, respectively).

\section{Discussion}

Endothelial dysfunction precedes the development of plaques in animal models and develops initially in humans at coronary branch points, the same sites at which advanced plaques are later formed. ${ }^{19}{ }^{20}$ Thus endothelial dysfunction is thought to be the initial event in a process that culminates in atherosclerosis. ${ }^{5}$ This study showed that two indices of endothelial function, endothelium dependent dilatation and plasma von Willebrand factor concentration, were abnormal in patients with chronic renal failure when compared with healthy subjects. These abnormalities were independent of differences in known vascular risk factors and vessel size between the two groups and suggest that chronic renal failure may directly induce endothelial dysfunction, thereby promoting the development of atherosclerosis.

The finding that endothelial function is equally impaired in renal failure patients with and without clinically evident atherosclerotic vascular disease lends support to the suggestion that endothelial dysfunction precedes the development of atheroma in renal failure. It is also possible that clinically silent atheroma is present in most patients with renal failure by the time of presentation.

Previous studies examining endothelial function in chronic renal failure have shown a significant reduction in endothelium dependent dilatation and raised concentrations of several endothelium derived proteins including endothelin-1, thrombomodulin, and von Willebrand factor. ${ }^{12-14}{ }^{21-23}$ However, the subjects in these trials were either patients with advanced renal impairment (median glomerular filtration rates of $<25 \mathrm{ml} / \mathrm{min}$ ) or those receiving renal replacement therapy. This present study shows that endothelial function is equally impaired in patients with biochemically mild renal failure and in those with more advanced renal disease, suggesting that endothelial dysfunction is already present early in progressive renal failure. Further studies are required to assess endothelial function in a larger number of individuals with glomerular filtration rates of between 50 and $80 \mathrm{ml} / \mathrm{min}$ to determine the level at which endothelial dysfunction is induced. However, the Cockroft formula is a poor measure of glomerular filtration rate in patients with preserved renal function, and investigation of endothelial function in the mildest or subclinical forms of renal failure would require a more accurate estimate of the degree of renal impairment.

Nitric oxide regulates vessel tone, inhibits platelet activation, adhesion, and aggregation, inhibits smooth muscle proliferation, and modulates endothelial cell-leucocyte interactions. ${ }^{24-26}$ Impaired bioavailability of nitric oxide, as suggested by the reduction in endothelium dependent dilatation in patients with chronic renal failure, may contribute to the pathogenesis of atherosclerosis in uraemia. Although the precise mechanisms are unknown, uraemic serum contains various substances that may either reduce endothelial production of nitric oxide or promote nitric oxide breakdown, including asymmetrical dimethylarginine, ${ }^{27}$ homocysteine, ${ }^{24}{ }^{28}$ and oxidatively modified low density lipoproteins. ${ }^{29}$

Prevention and treatment of vascular disease is currently targeted at renal patients with advanced disease; however, our study would suggest that these strategies should be directed at patients at earlier stages of progressive renal failure before irreversible endothelial damage occurs. This is particularly important, as moderate renal dysfunction is surprisingly common $-8 \%$ of 6000 middle aged individuals from the Framingham population were found to have mild renal insufficiency (serum creatinine 120 to $265 \mu \mathrm{mol} / \mathrm{l}) .^{30}$ In up to half of all patients, the incidence of coronary artery disease cannot be explained by conventional risk factors. Therefore the study of the aetiology of coronary artery disease in patients with renal failure may provide valuable insights into mechanisms in the general population.

In conclusion, we have shown in this study that endothelial function is abnormal in chronic renal failure, even in patients with biochemically mild renal insufficiency and in those without clinically evident atherosclerotic vascular disease. This suggests that uraemia may directly promote the development of atherosclerosis early in the progression of chronic renal failure. This work was funded by a project grant from the British Heart
Foundation. JT and JNT are supported by the British Heart Foundation. JT and JNT are supported by the British Heart
Foundation. We would like to thank the patients, doctors, and Foundation. We would like to thank the patients, doctors, and
staff at both the Queen Elizabeth Hospital Renal Unit and the staff at both the Queen Elizabeth Hospital Renal Unit and the
Lordswood Road Surgery, Birmingham, for all their help in enabling us to complete this study. We would also like to thank the Department of Rheumatology, University of Birmingham, for measuring plasma von Willebrand factor levels.

1 Jungers $\mathrm{P}$, Massy ZA, Khoa TN, et al. Incidence and risk factors of atherosclerotic cardiovascular accidents in predifactors of atherosclerotic cardiovascular accidents in predialysis chronic renal failure patients: a prosp
Nephrol Dial Transplant 1997;12:2597-602.

2 Kasiske BL, Guijarro C, Massy ZA, et al. Cardiovascular disease after renal transplantation. $\mathcal{F}$ Am Soc Nephrol 1996; 7:158-65

3 Raine AE, Margreiter R, Brunner FP, et al. Report on management of renal failure in Europe, XXII, 1991. Nephrol Dial Transplant 1992;2:7-35.

4 Excerpts from the USRDS 1996 annual data report. Am $\mathcal{F}$ Kidney Dis 1996;28:S79-102.

5 Ross R. The pathogenesis of atherosclerosis: a perspective for the 1990s. Nature 1993;362:801-9.

6 Celermajer DS, Sorensen KE, Gooch VM, et al. Noninvasive detection of endothelial dysfunction in children and adults at risk of atherosclerosis. Lancet 1992;340:111115.

7 Celermajer DS, Sorensen KE, Bull C, et al. Endotheliumdependent dilation in the systemic arteries of asymptomatic subjects relates to coronary risk factors and their interaction. $\mathcal{F} \mathrm{Am}$ Coll Cardiol 1994;24:1468-74.

8 Anderson TJ, Uehata A, Gerhard MD, et al. Close relation of endothelial function in the human coronary and peripheral circulations. F Am Coll Cardiol 1995;26:1235-41.

9 Sadler JE. von Willebrand factor. F Biol Chem 1991;266: 22777-80.

10 Meade TW, Cooper JA, Stirling Y, et al. Factor VIII, ABO blood group and the incidence of ishaemic heart disease. $\mathrm{Br}$ F Haematol 1994;88:601-7.

11 Boneu B, Abbal M, Plante J, et al. Factor-VIII complex and endothelial damage [letter]. Lancet 1975;i:1430.

12 Takagi M, Wada H, Mukai K, et al. Increased vascular endothelial cell markers in patients with chronic renal failure on maintenance haemodialysis. Blood Coagul Fibrinolysis 1994;5:713-17. 
13 van Guldener C, Lambert J, Janssen MJ, et al. Endotheliumin chronic haemodialysis patients. Nephrol Dial Transplant in chronic haemo $1997 ; 2: 14-18$.

14 van Guldener C, Janssen MJ, Lambert J, et al. No change in impaired endothelial function after long-term folic acid therapy of hyperhomocysteinaemia in haemodialysis patients. Nephrol Dial Transplant 1998;13:106-12.

15 Joki N, Hase H, Nakamura R, et al. Onset of coronary artery disease prior to initiation of haemodialysis in patients with end-stage renal disease. Nephrol Dial Transplant 1997;12 $718-23$.

16 Cockcroft DW, Gault MH. Prediction of creatinine clearance from serum creatinine. Nephron 1976;16:31-41.

17 Meredith IT, Currie KE, Anderson TJ, et al. Postischemic vasodilation in human forearm is dependent on endothelium-derived nitric oxide. Am f Physiol 1996;270: H1435-40.

18 Joannides R, Haefeli WE, Linder L, et al. Nitric oxide is responsible for flow-dependent dilatation of human periphresponsible for flow-dependent dilatation of human periph-

19 McLenachan JM, Vita J, Fish DR, et al. Early evidence of endothelial vasodilator dysfunction at coronary branch points. Circulation 1990;82:1169-73.

20 Harrison DG, Armstrong ML, Freiman PC, et al. Restoration of endothelium-dependent relaxation by dietary treatment of atherosclerosis. $\mathcal{F}$ Clin Invest 1987;80:1808-11

21 Kari JA, Donald AE, Vallance DT, et al. Physiology and biorenal failure. Kidney Int 1997;52:468-72.
22 Kuriyama S, Tomonari H, Yoshida H, et al. Endothelial cell dysfunction in patients with impaired renal function [In Japanese]. Fpn f Nephrol 1996;38:372-8.

23 Haaber AB, Eidemak I, Jensen T, et al. Vascular endothelial cell function and cardiovascular risk factors in patients with chronic renal failure. $\mathcal{F}$ Am Soc Nephrol 1995;5:1581-4.

24 Upchurch GR, Welch GN, Loscalzo J. Homocysteine, EDRF, and endothelial function. $\mathcal{F}$ Nutr 1996;126(suppl 4):1290-4S.

25 Murad F, Arnold WP, Mittal CK, et al. Properties and regulation of guanylate cyclase and some proposed functions for cyclic GMP. Adv Cyclic Nucleotide Res 1979;11:175-204.

26 Luscher TF, Tanner FC, Noll G. Lipids and endothelial function: effects of lipid-lowering and other therapeutic interventions. Curr Opin Lipidol 1996;7:234-40.

27 Vallance P, Leone A, Calver A, et al. Accumulation of an endogenous inhibitor of nitric oxide synthesis in chronic renal failure. Lancet 1992;339:572-5.

28 Stamler JS, Osborne JA, Jaraki O, et al. Adverse vascular effects of homocysteine are modulated by endotheliumderived relaxing factor and related oxides of nitrogen. $\mathcal{F}$ Clin Invest 1993;91:308-18.

29 Chin JH, Azhar S, Hoffman BB. Inactivation of endothelial derived relaxing factor by oxidized lipoproteins. $7 \mathrm{Clin}$ Invest 1992;89:10-18.

30 Culleton BF, Larson MG, Wilson PWF, et al. Prevalence and outcomes of mild renal insufficiency in the community [abstract]. F Am Soc Nephrol 1998;9(suppl):145. 